\title{
ГЕОФИЗИКА
}

УДК 550.831

DOI: https://doi.org/10.17308/geology.2021.2/3489

ISSN 1609-0691

Поступила в редакцию: 01.03.2021

Принята к публикации: 20.05 .2021

Опубликована онлайн: 30.06 .2021

\section{Влияние атмосферного давления на результаты измерений силы тяжести}

\author{
(C)2021 Ю. В. Антонов ${ }^{\circledR}$ \\ Воронежский государственный университет, \\ Университетская пл., 1, 394018, Воронеже, Российская Федерация
}

\begin{abstract}
Аннотация
Bведение: Приведены результаты сравнения атмосферного давления и неприливных вариаций силы тяжести в г. Бишкек (Кыргызстан) в год спокойного Солнца (2018 г.). Дело в том, что если изменение давления невелико, то в год спокойного Солнца, когда космические помехи минимальны, оценка влияния давления будет более надежной.

Методика: Для обработки данных были выбраны отрезки измерений с наибольшей амплитудой изменения давления, с наименышим количеством землетрясений и с отсутствием пульсаций силы тяжести. Из исходных данных были исключены линейный тренд, связанный со смещением нульпункта, и значения лунно-солнечных вариаций. Для удобства сравнения давление пересчитано в условные единицы в размерности миллигалов.

Обсуждение результатов: Влияние давления за исключением экстремальных случаев минимально, и учет его влияния надо делать в зависимости от конкретных условий. Установлен факт, что экстремумы атмосферного давления соответствуют экстремумам градиента лунно-солнечных вариаций. Причина такой корреляции на данном этапе труднообъяснима. Но одно очевидно, что это связано с деформацией атмосферы за счет Луны и Солнца.

Ключевые слова: атмосферное давление, лунно-солнечные вариации силы тяжести; неприливные вариации силы тяжести; метод осреднения

Для цчитирования: Антонов Ю. В. Влияние атмосферного давления на результаты измерений силы тяжести // Вестник Воронежского государственного университета. Серия: Геология. 2021. №2. С. 64-70. DOI: https://doi.org/10.17308/geology.2021.2/3489
\end{abstract}

\section{Введение}

Как показывают исследования [1-5], неприливные вариации силы тяжести во времени осложняются лунносолнечными вариациями, землетрясениями, ударами корональных выбросов масс по магнитосфере и метеорных потоков по атмосфере Земли и т. д. Год 2018 пришелся на год спокойного Солнца, когда воздействие космических факторов на неприливные вариации оказались минимальными. Поскольку влияние космических факторов минимальное, то была принята попытка оценить влияние атмосферного давления, так как в принципе оно (давление) невелико, и при отсутствии излишних внешних помех легче получить более достоверную оценку.

\section{Исходные данные}

Для оценки использованы мониторинговые наблюдения силы тяжести во времени, выполненные на Бишкекском геодинамическом полигоне РАН (г. Бишкек, Киргизстан) и данные об атмосферном давлении с метеостанции в аэропорту г. Бишкека [6]. Измерения силы тяжести проводились гравиметром CG - 5 AUTOGRAV на базе, которая расположена в 30 км за городом, так что технические помехи практически исключены. Гравиметр находится в штольне с постоянной температурой (круглый год около $8^{\circ} \mathrm{C}$ ). Прибор позволяет измерять силу тяжести и наклоны по двум ортогональным осям. Одновременно программное

Контент доступен под лицензией Creative Commons Attribution 4.0 License.

\footnotetext{
Антонов Юрий Васильевич, e-mail: yuriyantonov@yandex.ru
} 
обеспечение прибора позволяет вычислять теоретические значения силы тяжести от Луны и Солнца, которые вычитаются из наблюденных значений. Оставшуюся часть измерений, условно назовем неприливной вариацией. Под неприливными вариациями силы тяжести будем понимать все изменения силы тяжести, исключая лунно-солнечные вариации, вне зависимости от источника их происхождения.

\section{Методика обработки гравиметрических измерений}

Вычисление неприливных вариаций силы тяжести делается следующим образом. На первом этапе исключается притяжение Луны и Солнца. В связи с тем, что в программном продукте гравиметра CG - 5 AUTOGRAV, в котором рассчитывается притяжение Луны и Солнца, обнаружены погрешности [1-4], то лунно-солнечные вариации силы тяжести заново рассчитывались по программе Тиммена и учитывались при обработке [8, 9]. После этого по исправленным значениям определяется линейный тренд, который исключается. Значения тренда в основном отражают в себе сползание нульпункта прибора и какую-то низкочастотную часть изменения внешнего гравитационного поля. Как показывают исследования [1, 2], неприливная вариация с помощью метода осреднения делится на локальную (остаточную) и осредненную неприливные вариации. Кстати, локальная неприливная вариация может быть получена с помощью метода осреднения непосредственно из исходных данных, минуя операции учета лунно-солнечных вариаций силы тяжести и смещения нуль-пункта прибора. Вариация, полученная по исходным наблюдениям с помощью осреднения, и вариация силы тяжести, полученная обычным способом, практически совпадают между собой. Разность между ними не превышает одного микрогала, что минимум на два порядка меньше амплитуды наблюдаемых неприливных вариаций и погрешности измерений $[1,2]$. Так что в большинстве случаев для получения неприливной вариации лучше пользоваться методом осреднения.

Значения силы тяжести измерялись с дискретностью 1 мин. Значения атмосферного давления приведены с интервалом 3 часа [6]. Для удобства сравнения давления с данными силы тяжести проведена интерполяция значений давления с дискретностью 1 мин. Давление меняется достаточно линейно, следовательно, интерполяция между временами замеров не вносит дополнительных помех.

Вопрос об учете влияния атмосферного давления при гравиметрических измерениях ранее рассматривалось в [7]. Там же [7] приводится приближенная формула перевода давления в значения силы тяжести:

$$
\Delta \mathrm{g}=\mathrm{K} \Delta \mathrm{P},
$$

где $\Delta \mathrm{g}$ - приращение силы тяжести; $\Delta \mathrm{P}$ - приращение атмосферного давления; К - коэффициент перевода значений давления в значения силы тяжести. Этот коэффициент равен $0,056810^{-3}$ для давления в мм рт. ст. или $0,042610^{-3}$ при давлении в миллибарах. Значения атмосферного давления для удобства сравнения с неприливными вариациями переведены с помощью указанной формулы в условные значения силы тяжести. Кроме того, из значений атмосферного давления вычтена постоянная и оставлена вариативная часть, которая собственно оказывает влияние на значения силы тяжести.

\section{Обсуждение результатов интерпретации}

Рассмотрим некоторые особенности морфологии графиков изменения давления и силы тяжести. На рис. 1 приведены графики суточного хода силы тяжести (рис. $1, a)$, давления (рис.1, $b$ ) и остаточной неприливной вариации силы тяжести (рис.1, c). На остаточной неприливной вариации четко выделяется пульсация силы тяжести (15-18 января) [2], которая образуется ударами по земной атмосфере метеорных потоков (альфа Круциды и дельта Канкриды), зенитное число (количество вспышек в час) которых не превышает 5 единиц. Никакой корреляции неприливной пульсации силы тяжести с кривой изменения давления, а тем более с суточным ходом силы тяжести, нет.

Примерно также ведут себя графики давления, суточного хода силы тяжести и остаточная аномалия в августе того же года (рис. 2). Но в данном случае есть одна существенная деталь: нет пульсаций силы тяжести в остаточной неприливной вариации (рис. 2, c) или, по крайней мере, еле заметны только для опытного глаза. Но в ночь на 12 августа наблюдался известный метеорный ливень Персеид с зенитным числом более 120 единиц. Каких-либо заметных изменений на графиках давления и суточного хода силы тяжести не отмечено.

Но главное то, что отсутствуют пульсации силы тяжести, которые образуются при ударе метеорного потока об атмосферу Земли $[1,2]$. Дело в том, что в астрономии принято оценивать мощность метеорных потоков по числу вспышек за час (зенитное число), а вспышку дают только крупные частицы. Но основную массу потока часто создают более мелкие частицы, которые при ударе по атмосфере обеспечивают дополнительные колебания. Кроме того, изменение плотности метеорного потока можно объяснить изменением геометрических размеров потоков во времени. Метеорные потоки в момент рождения условно можно представить в виде «пыльного тора» с переменной плотностью пыли. Со старением потока размеры потока увеличиваются, а плотность потока уменьшается. В конкретном случае диаметр потока Персеиды составляет около 80 млн. км, а у Круцидов и Канкридов на порядок меньше. Определение же размеров метеорных потоков вещь ненадежная и спорная. Грубо о размерах можно судить по длительности неприливных пульсаций силы тяжести.

На рис. 3 приведены периодограммы частот для неприливных вариаций при наличии пульсаций и без. Вариации выбраны для времени, когда отсутствовали какие-либо помехи (особенно землетрясения). Частотные характеристики неприливных вариаций идентичны и укладываются в отрезок времени от 2 до 20 мин, что 


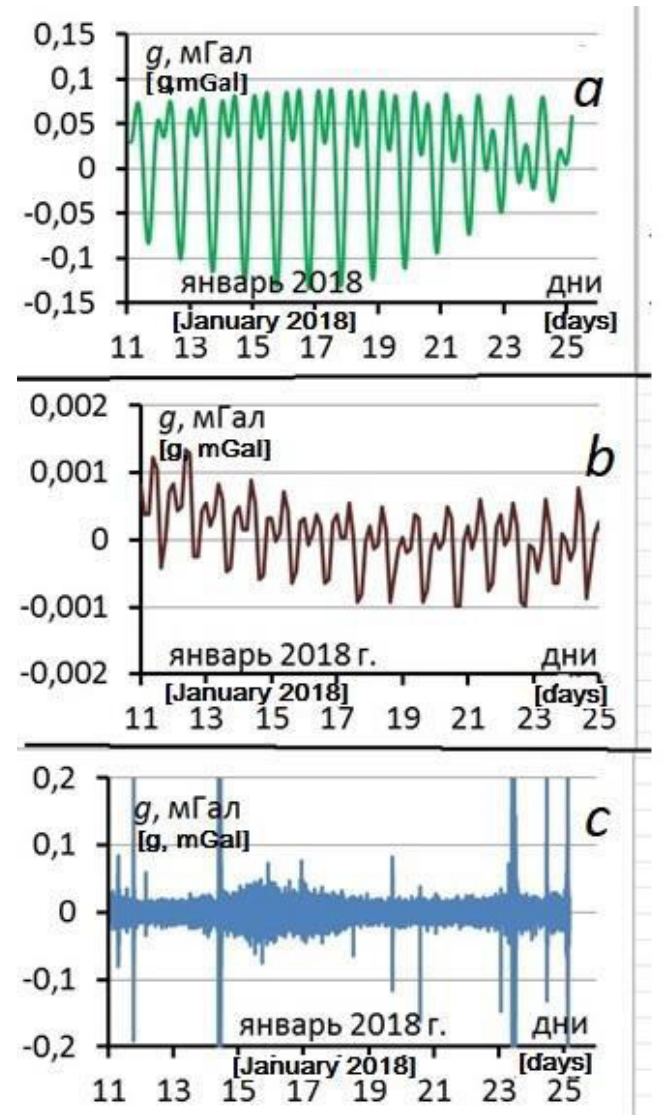

Рис. 1. Изменения лунно-солнечных вариации силы тяжести и атмосферного давления при наличии пульсаций силы тяжести: $a$ - график лунно-солнечных вариаций силы тяжести; $b$ - локальные изменения атмосферного давления; с остаточная неприливная вариация силы тяжести.

[Fig. 1. Change in lunar-solar gravity variations and atmospheric pressure in the presence of gravity pulsations: $(a)$ diagram of lunar-solar gravity variations; $(b)$ local changes in atmospheric pressure; (c) the residual non-tidal gravity variations.]
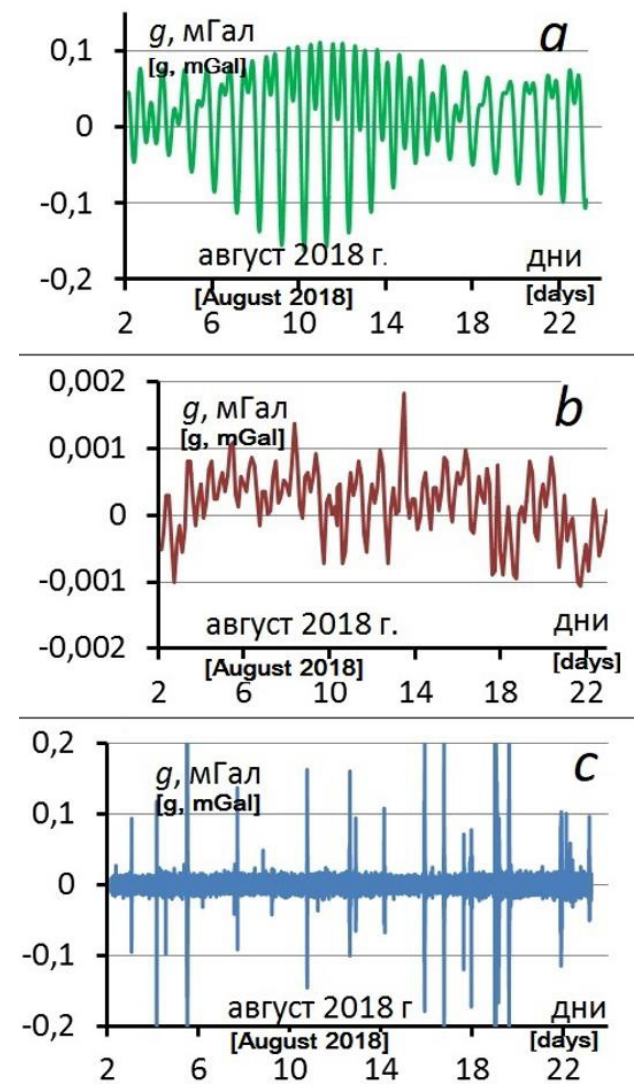

Рис. 2. Изменение лунно-солнечных вариации силы тяжести и атмосферного давления при отсутствии пульсаций силы тяжести. То же самое, что на рис. 1

[Fig. 2. Change in lunar-solar gravity variations and atmospheric pressure in the absence of gravity pulsations. The same as in Fig.1.]

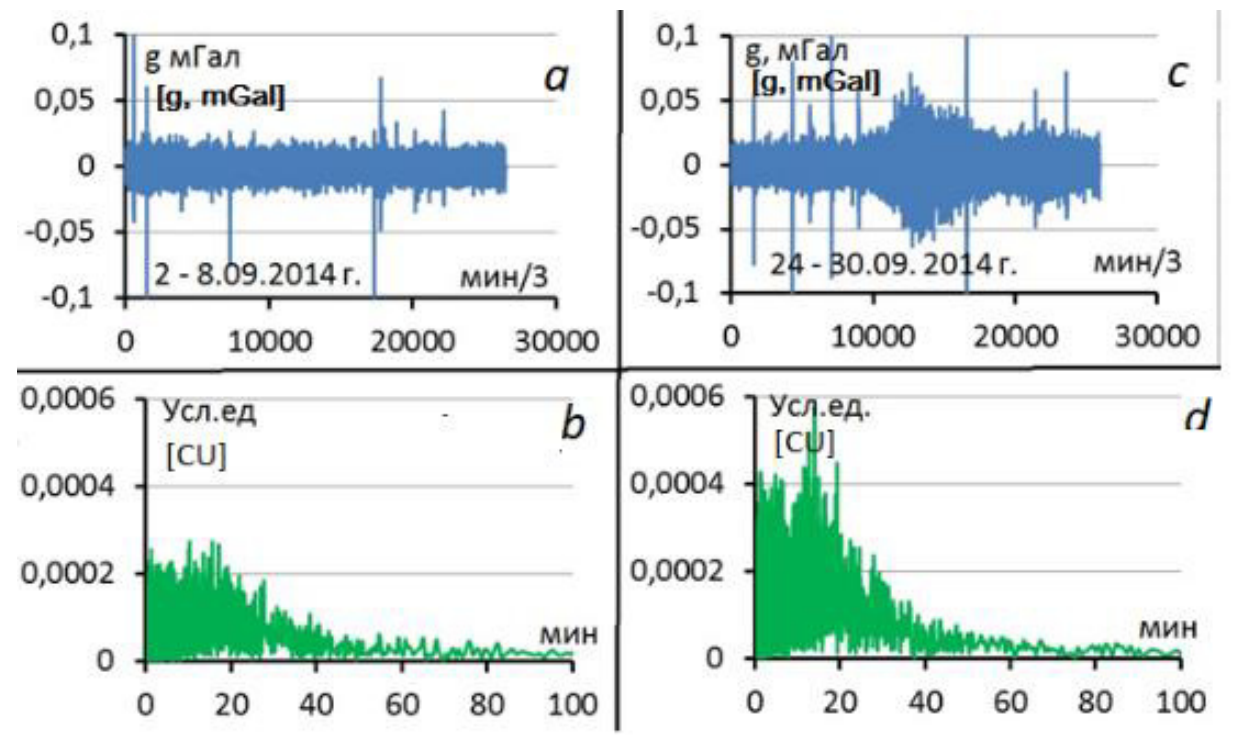

Рис. 3. Периодограммы остаточной вариации силы тяжести при наличии и отсутствии пульсаций силы тяжести: $a-$ остаточная неприливная вариация силы тяжести при отсутствии пульсаций; $b$ - периодограмма остаточной вариации силы тяжести при отсутствии пульсаций; $c$ - остаточная неприливная вариация силы тяжести при наличии пульсаций; $d$ - периодограмма остаточной вариации силы тяжести при наличии пульсаций. 
[Fig. 3. Periodograms of the residual gravity variations in the presence and absence of gravity pulsations: $(a)$ the residual nontidal gravity variations in the absence of pulsations; $(b)$ periodogram of the residual gravity variations in the absence of gravity pulsations; (c) the residual non-tidal gravity variations in the presence of gravity pulsations; $(d)$ periodogram of residual gravity variations in the presence of gravity pulsations.]

что полностью согласуется с выводами о собственных колебаниях атмосферы [5]. Правда, при наличии пульсации амплитуды спектров в два раза больше.

Вернемся к графикам лунно-солнечных вариаций и атмосферного давления (рис. 1, 2). Внешне они похожи, но посмотрим эти графики в увеличенном мас- штабе (рис. 4). Если сравнивать лунно-солнечные вариаций и давления (рис. $4, a, c$ ), то при внимательном рассмотрении видим, что максимумы давления больше тяготеют к максимальным значениям градиента лунносолнечных вариаций. Поэтому было решено продифференцировать лунно-солнечные вариации и сравнить с давлением. Налицо полное совпадение изменения производной силы тяжести и давления (рис. $4, b, d)$. На графиках производная (рис. $4, b, d$ ) заменена приращением вариаций $\delta \mathrm{g}=\Delta \mathrm{g}\left(\mathrm{t}_{\mathrm{n}+1}\right)-\Delta \mathrm{g}\left(\mathrm{t}_{\mathrm{n}}\right)$. с шагом 1 мин. Здесь сталкиваемся с такой же ситуацией, которую имеем между силой тяжести и вертикальной составляющей сейсмического поля [3].

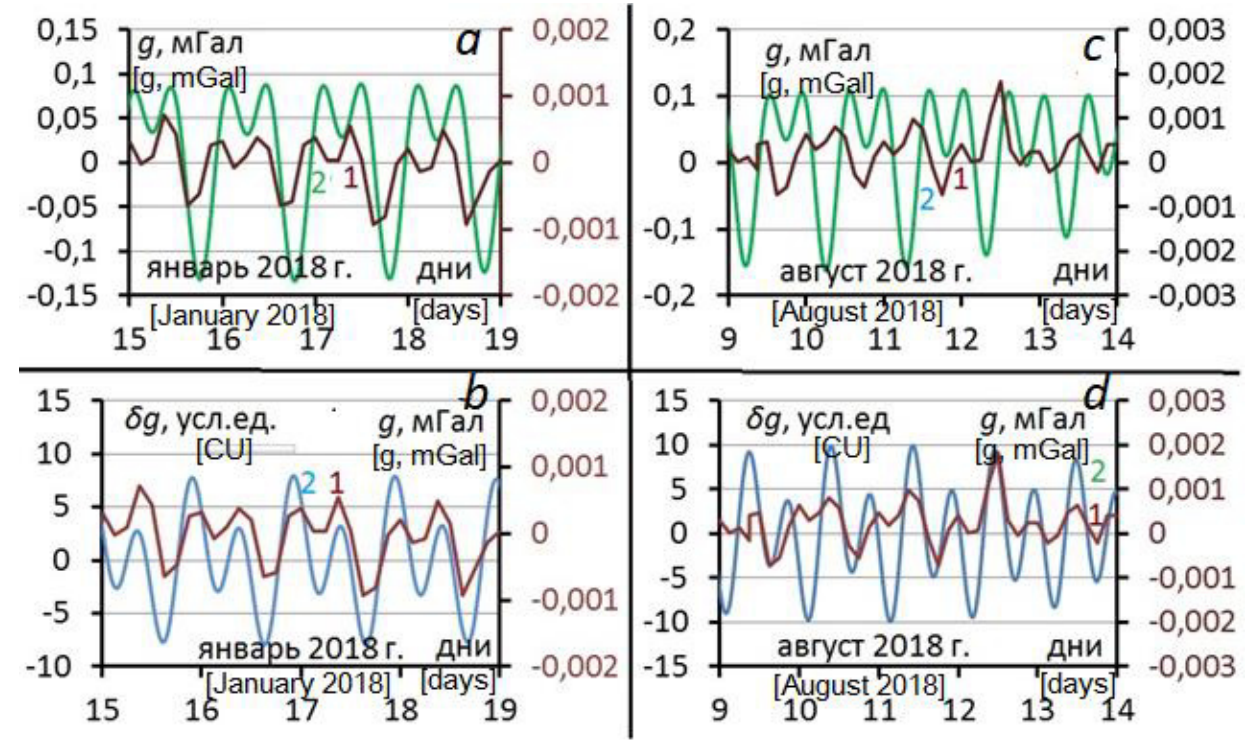

Рис. 4. Сравнение графиков лунно-солнечных вариации силы тяжести и их градиента во времени с графиками атмосферного давления: $a-$ графики лунно-солнечных вариаций силы тяжести (кривая 2) и атмосферного давления (кривая 1) в январе 2018 г.; $b$ - график градиента лунно-солнечных вариаций силы тяжести (кривая 2) и график атмосферного давления (кривая 1) в январе 2018 г.; $c, d-$ те же обозначения для августа 2018 г.

[Fig. 4. Comparison of charts of the lunar-solar gravity variations and their gradient in time with atmospheric pressure graphs: (a) graphs of lunar-solar gravity variations (curve 2) and atmospheric pressure (curve 1) in January 2018; (b) graph of the gradient of the lunarsolar gravity variations (curve 2) and the graph of atmospheric pressure (curve 1) in January 2018; (c, $d$ ) the same for August 2018.]

Как видим из рис. 1-4, поправки за влияние давления невелики и варьируют в пределах одного мкГала, что соответствует погрешности измерений современных гравиметров. Тем не менее, наблюдается четкая корреляция между давлением и лунно-солнечными вариациями силы тяжести. В случае, когда поправка за давление превышает погрешность измерений в три и более раз, то соотношение между производной остается тем же (рис. 5, a). Кстати, здесь же (рис. 5, $b$ ) приведена неприливная вариация силы тяжести, на которой проявляется землетрясение резким изменением силы тяжести до \pm 0.5 мГал. Время события совпадает с резким изменения давления и наличием сильнейшего ливня [7]. Вообще-то, в Средней Азии все климатические катаклизмы (ливни, пылевые бури и т.д.) чаще всего сопровождаются землетрясениями. Видимо, резкое изменение давления в альпийской активизированной зоне является достаточной силой, чтобы служить «спусковым крючком» для создания землетрясения. Но данный вопрос очень сложен и требует отдельного обсуждения.
В силу малости изменения поправок за счёт давления при проведении гравиметрических работ их можно не учитывать, за исключением случаев, когда поправки могут достигать 3-5 мкГал. Это случаи высокоточных работ в инженерной или подземной гравиразведке. А вот при проведении измерений в горной местности поправки за давление надо вводить, поскольку превышение в 10 м дает уже величину почти 5 мкГал.

Что же касается мониторинговых наблюдений здесь все сложнее. Обратимся к рис. 6, на котором приведены результаты вычисления остаточной неприливной вариации силы тяжести (рис. 6, c) с помощью метода осреднения [1]. Осредненная неприливная вариация (рис. $6, a)$ и кривая давления (рис. $6, b$ ) в целом совпадают между собой. Совпадение чётко видно в увеличенном масштабе (рис. $6, d$ ). Тогда при вычислении неприливных вариаций с помощью метода осреднения влияние давления автоматически исключается. Но сразу возникает другой вопрос. Чем можно объяснить природу осредненной вариации? Осредненная неприливная вариация (рис. $6, a)$, по амплитуде на порядок больше 
влияния давления, но по морфологии совпадает с давлением. На данный момент у автора нет ответа.

\section{Заключение}

Таким образом, по результатам исследования можно уверенно сказать, что лунно-солнечные вариации силы тяжести и изменения атмосферного давления тесно связаны между собой. Атмосфера окружает Землю, поэтому влияние всех внешних и внутренних факторов находит отклик в атмосфере, тем самым определяя состояние, как самой атмосферы, так и состояние геофизических полей внутри и вне её. Отдельные всплески изменения давления, скорее всего, объясняются процессами, происходящими в нижней части атмосферы.

Рис. 5. Сравнение аномалии атмосферного давления и неприливной вариации силы тяжести: $a$ - графики лунно-солнечных вариаций силы тяжести (кривая 2) и атмосферного давления (кривая 1) в январе 2018г.; $b$ - неприливная вариация силы тяжести.

[Fig. 5. Comparison of atmospheric pressure anomalies and nontidal gravity variations: (a) graphs of lunar-solar gravity variations (curve 2) and atmospheric pressure (curve 1) in January 2018; (b) non-tidal gravity variation.]
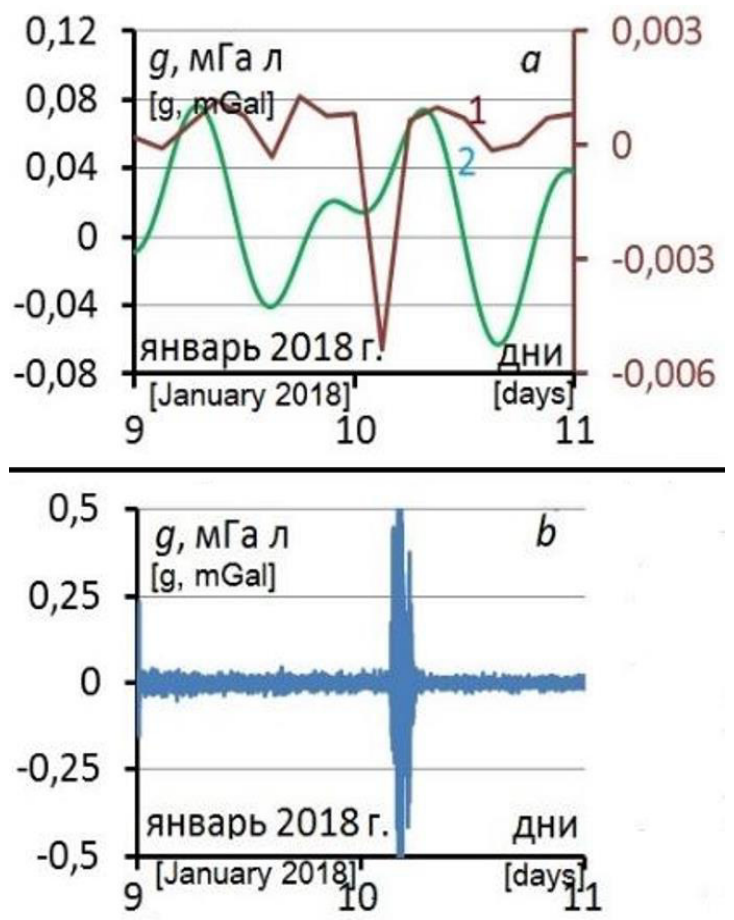
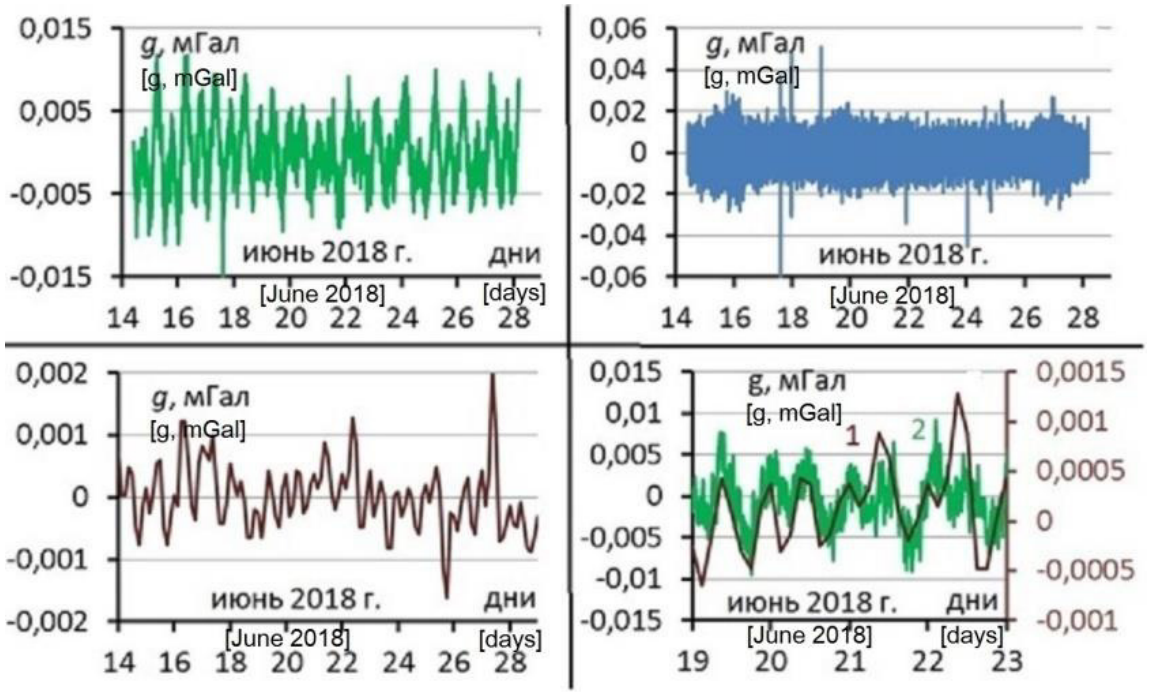

Рис. 6. Сравнение графиков осредненной неприливной вариации силы тяжести и атмосферного давления: $a$ - осредненная неприливная вариация силы тяжести; $b-$ график атмосферного давления; $c$ остаточная неприливная вариация силы тяжести; $d-$ сравнение осредненной неприливной вариации силы тяжести (кривая 2) с атмосферным давлением (кривая 1) [Fig. 6. Comparison charts of the average non-tidal gravity variations and atmospheric pressure: (a) average non-tidal gravity variations; $(b)$ graph of atmospheric pressure; $(c)$ residual non-tidal gravity variation; $(d)$ comparison of the average non-tidal gravity variations (curve 2) with atmospheric pressure (curve 1).
Конфликт интересов: Автор декларирует отсутствие явных и потенциальных конфликтов интересов, связанных с публикацией настоящей статьи.

\section{ЛИТЕРАТУРА}

1. Антонов Ю. В. Разделение неприливных вариаций силы тяжести на основе спектрального анализа и метода осреднения // Вестник Воронежского государственного университета. Сeрия: Геология. 2016. № 2. С. 100-106.

2. Антонов Ю. В., Сизаск И. А. Синхронные пульсации в неприливных вариациях гравитационного и сейсмического полей // Известия высших учебных заведений. Геология и разведка. 2015. №5. C. 46-52. DOI: https://doi.org/10.32454/0016-77622015-5-46-52

3. Антонов Ю. В., Антонова И. Ю. Сравнение приливных вариаций силы тяжести и вертикальной составляющей сейсмографа // Геофизика. 2013. №2. С. 27-31.
4. Антонов Ю. В. Влияние атмосферного фронта на показания гравиметров и сейсмометров // Известия высших учебных заведений. Геология и разведка. 2017. №4. С. 66-71.

5. Дикий Л. А. Теория колебаний земной атмосферы Л.: Гидрометеоиздат, 1969. $195 \mathrm{c.}$

6. Расписание погоды [Электронный ресурc]. URL: http://rp5.ru (дата обращения: 01.09.2020)

7. Бычков С. Г., Долгаль А. С., Симанов А.А. Вычисление аномалий силы тяжести при высокоточных гравиметрических съемках. Вопросы теории и практики геологической интерпретаџии геофизических полей. Материалы 43-й сессии Международного научного семинара им. Д. Г. Успенского. 2016 Пермь. УрО РАН. C. 39-42.

8. Boy J.-P., Hinderer J., Gegout P. Global atmospheric loading and gravity // Phys. Earth Planet. Inter. 1998. V. 109. P. 161-177.

9. Timmen L., Wenzel H.-G. Worldwide synthetic gravity tide parameters available on Internet // Bulletin d'information-Bureau gravimétrique international. 1994. V. 75. P. 32-40. 


\title{
Effect of atmospheric pressure on the results of gravity measurement
}

\author{
C)2021 Yu. V. Antonov ${ }^{凶}$ \\ Voronezh State University, \\ 1 Universitetskaya pl., Voronezh 394018, Russian Federation
}

\begin{abstract}
Introduction: The article presents the results of comparing the atmospheric pressure and non-tidal gravity variations in Bishkek (Kyrgyzstan) in a year solar minimum. (2018). If the pressure differences are insufficient, then in a year of solar minimum, when the cosmic disturbances are minimal, the assessment of the pressure influence will be more reliable.

Methodology: For data processing we selected measurement intervals with the greatest amplitude of pressure change, with the fewest earthquakes, and with no gravity pulsations. A linear trend associated with a shift of the zero point and the values of the lunar-solar gravity variations were excluded from the initial data. For ease of comparison, the pressure was expressed in conventional units of milligal dimension. Results and Discussion: The effect of pressure with the exception of extreme cases is minimal, and it should be taken into account depending on the specific conditions. It was established that the extremes of atmospheric pressure correspond to the gradient extremes of the lunar-solar gravity variations. The reason for this correlation is difficult to explain at this stage. However, one thing is clear: this is due to the atmosphere deformation caused by the Sun and the Moon.

Key words: atmosphere pressure, lunar-solar gravity variations; non-tidal gravity variations, method of averaging.

For citation: Antonov Yu. V. Effect of atmospheric pressure on the results of gravity measurement. Vestnik Voronezhskogo gosudarstvennogo universiteta. Seriya: Geologiya - Proceedings of Voronezh State University. Series: Geology, 2021, no. 2, pp. 64-70. DOI: https://doi.org/10.17308/geology.2021.2/3489

Conflict of interests: The author declares the absence of obvious and potential conflicts of interest related to the publication of this article.
\end{abstract}

\section{REFERENCE}

1. Antonov Yu. V. Razdelenie neprilivnykh variatsii sily tyazhesti na osnove spektral'nogo analiza i metoda osredneniya [Separation of non-tidal gravity variations based on spectral analysis and averaging method]. Vestnik Voronezhskogo gosudarstvennogo universiteta. Seriya: Geologiya - Proceedings of Voronezh State University. Series: Geology, 2013, no. 2, pp. 100-106. (In Russ.).

2. Antonov Yu. V., Sizask I. A. Sinkhronnye pul'satsii v neprilivnykh variatsiyakh gravitatsionnogo $i$ seismich- eskogo polei [Synchronous pulsations in non-tidal variations of gravitational and seismic fields]. Izvestiya vysshikh uchebnykh zavedenii. Geologiya i razvedka-Proceedings of higher educational establishments. Geology and Exploration, 2015, no. 5, pp. 46-52. (In Russ.).

3. Antonov Yu. V., Antonova I. Yu. Sravnenie prilivnykh variatsii sily tyazhesti i vertikal'noi sostavlyayushchei seismografa [Comparison of tidal variations of gravity and vertical component of the seismograph]. Geofizika-Geophysics, 2016, no. 2, pp. 27-31. (In Russ.).

The content is available under Creative Commons Attribution 4.0 License.

\footnotetext{
Yuriy V. Antonov, e-mail: yuriyantonov@yandex.ru
} 
4. Antonov Yu. V. Vliyanie atmosfernogo fronta na pokazaniya gravimetrov i seymometrov [Influence of the atmospheric front on the readings of gravimeters and seismometers]. Izvestiya vysshikh uchebnykh zavedenii. Geologiya i razvedka-Proceedings of higher educational establishments. Geology and Exploration, 2017, no. 4, pp. 66-71. (In Russ.) DOI: https://doi.org/10.32454/00167762-2017-4-66-71

5. Dikii L. A. Teoriya kolebanii zemnoi atmosfery [Theory of oscillations of Earth's atmosphere]. Leningrad, Gidrometeoizdat publ., 1969, 195 p. (In Russ.).

6. Raspisanie pogody [Weather schedule]. Available at: http://rp5.ru archive (accessed 14 March 2021)

7. Bychkov S. G., Dolgal' A. S., Simanov A.A. Vychislenie anomalii sily tyazhesti pri vysokotochnykh gravimetri- cheskikh s"emkakh. Voprosy teorii i praktiki geologicheskoi interpretatsii geofizicheskikh polei. [Calculation of gravity anomalies in high-precision gravimetric surveys]. Materialy 43-i sessii Mezhdunarodnogo nauchnogo seminara im. D. G. Uspenskogo [Materials of the 43rd session of the International Scientific Seminar. D. G. Uspensky.]. Perm, 2016. 142 p. (In Russ.)

8. Boy J.-P., Hinderer J., Gegout P. Global atmospheric loading and gravity. Phys. Earth Planet. Inter, 1998, vol. 109, pp. 161-177.

9. Timmen L., Wenzel H.-G. Worldwide synthetic gravity tide parameters available on Internet. Bulletin d'information-Bureau gravimétrique international, 1994, vol. 75, pp. 32-40.
Антонов Юрий Васильевич - д. тех. н., профессор, Воронежский государственный университет, Воронеж, Российская Федерация; E-mail: yuriyantonov@yandex.ru; ORCID http://orcid.org/0000-0002-3323-9697

Автор прочитал и одобрил окончательный вариант рукописи.
Yuriy V. Antonov - Dr.habil. in Techn., Professor, Voronezh State University, Voronezh, Russian Federation; E-mail: yuriyantonov@yandex.ru; ORCID http://orcid.org/0000-0002-3323-9697

Author have read and approved the final manuscript. 